\title{
Complement and the recruitment of mononuclear osteoclasts
}

\author{
D C Mangham, D J Scoones, M T Drayson
}

\begin{abstract}
Aim-To investigate the hypothesis that complement mediates the recruitment of mononuclear osteoclast precursors to the exposed mineralised bone surface.

Methods-Synthetic hydroxyapatite was incubated in vitro with fresh human serum, with and without complement activation inhibitors. Assays for complement components and the generation of the C3 breakdown product C3d were done. C3 deposition in human fetal tibia primary spongiosa was localised immunohistochemically and complement receptors CR1, CR2, CR3, and CR4 were localised cellularly. Immunohistochemical and enzyme histochemical characterisation of the mononuclear and multinuclear osteoclasts was made with emphasis on their association with complement C3 deposition.
\end{abstract}

Results-Components of complement bind to synthetic hydroxyapatite crystals and, at lower concentrations, C3d was generated in the fluid phase. C3 was deposited in a focal and linear distribution on newly formed bone trabecular surfaces in the primary spongiosa. In a similar distribution CD61, CD68, and tartrate resistant acid phosphatase positive mononuclear osteoclasts were shown in close apposition to the bone trabecular surface. These mononuclear osteoclasts, unlike multinucleate osteoclasts, expressed the complement receptors $\mathrm{CR3}$ and CR4. CR1 and CR2, however, could not be shown on either mononuclear or multinuclear osteoclasts.

Conclusion-It is suggested that C3 deposition on mineralised bone surfaces mediates the recruitment of mononuclear osteoclasts to this site. As the mononuclear osteoclasts fuse to form the multinucleate osteoclast, complement receptor expression is lost.

( Clin Pathol 1993;46:517-521)

Osteoclasts are multinucleated cells which resorb bone. Osteoclasts attain their multinucleated form by fusion of mononuclear precursors. ${ }^{1}$ Studies on chick-quail chimera, ${ }^{23}$ parabiotic rats, ${ }^{4}$ and marrow transplantation in osteopetrotic rats $^{56}$ show that osteoclast precursors are circulating cells originating in the bone marrow. These precursors have not been identified, although they are believed to belong to the monocyte-macrophage lineage. In support of this, the immunophenotype of osteoclasts has been well characterised and shown to express many of the antigens of the monocyte-macrophage lineage. ${ }^{7}$ Mononuclear cells have been identified in histological sections of bone. These are closely applied to the bone surface and show a similar immunophenotype to that of the multinucleate osteoclasts. ${ }^{8}$

In vitro osteoblasts, in the presence of parathyroid hormone, can remove the unmineralised organic layer of osteoid to expose the mineralised bone beneath, and bone so modified has an increased susceptibility to osteoclastic resorption. ${ }^{9}$ Osteoblast cells produce collagenase and plasminogen activator, and production is increased by parathyroid hormone, 1,25 dihydroxycholecalciferol, and prostaglandin $\mathrm{E},{ }^{10} 11$ all factors that stimulate osteoclast bone resorption in vivo. Sato et al showed that the C3 component of complement can be produced by mouse osteoblasts and is important in the differentiation of bone marrow cells in osteoclasts. ${ }^{17}$

In this study we investigated the hypotheses that after the removal of osteoid by osteoblasts the exposed mineralised bone activates and binds complement, thus facilitating the recruitment of osteoclast precursors to the bone surface.

There is evidence to suggest that hydroxyapatite crystals in mineralised bone will bind the third component of the complement system (C3) on exposure to plasma. ${ }^{12}$ Our initial studies centre around in vitro experiments to clarify this issue. To assess the role of complement in vivo, frozen tissue sections of human fetal long bones were used. The primary spongiosa provides a dynamic view of bone cellular metabolism in that sequential stages of unidirectional bone growth are represented from the calcifying zone of hypertrophic chondrocytes, through the area of rapid bone deposition coupled to a lesser degree of osteoclasis in the primary spongiosa, to the increasingly quiescent region in the diaphysis. Using immunohistochemical means, linear and focal deposition of C3 was detected on exposed mineralised bone in the primary spongiosa. For C3 to be involved in the recruitment of osteoclast precursors, these cells must express complement receptors. Attention was focused on the expression of complement receptors by multinucleate osteoclasts and their mononuclear precursors. 
Table 1

\begin{tabular}{llll}
\hline & & \multicolumn{2}{l}{ Cells stained } \\
\cline { 3 - 4 } Antibody source & Antigen & $\begin{array}{l}\text { Multinucleate } \\
\text { osteoclasts }\end{array}$ & $\begin{array}{l}\text { Mononuclear } \\
\text { osteoclasts }\end{array}$ \\
\hline$\star$ & CD 11 1b & - & + \\
Binding site & CD 11c & - & + \\
$\star$ & CD 18 & - & + \\
$\begin{array}{l}\text { Binding site } \\
\text { Dako }\end{array}$ & CD 21 & - & - \\
Cako & CD 35 & - & - \\
Dako & CD 68 & + & + \\
& C3 & $\begin{array}{l}\text { Focal and linear staining of } \\
\text { the bone trabecular surface at }\end{array}$ \\
& & & the primary spongiosa \\
\hline
\end{tabular}

^Kindly supplied by $\mathrm{Dr}$ CM Bunce, Department of Immunology, Birmingham University.

All immunostaining was carried out on frozen sections (except antiCD68 on paraffin wax sections).

The latter were identified by their close application to the bone surface in the primary spongiosa, and their expression of markers of osteoclast differentiation.

\section{Methods \\ INCUBATION OF HYDROXYAPATITE CRYSTALS WITH FRESH HUMAN SERUM}

Fresh serum was obtained by venesection of healthy volunteers, allowing clotting to occur for 1 hour at room temperature before separation of serum by centrifugation. Hydroxyapatite crystals were obtained from Aldrich Chemical Company Inc. Catalogue No. 23,093-06. C3 and C4 were measured using a standard nephelometric technique. $\mathrm{C} 1 \mathrm{q}$, factor $B$, and $C 3 d$ were measured by TurboRID kits (Binding Site, Birmingham) and $\mathrm{CH} 50$ and $\mathrm{AP}-\mathrm{CH} 50$ measured by plate assay (Binding Site, Birmingham).

\section{IMMUNOHISTOCHEMISTRY AND ENZYME HISTOCHEMISTRY}

Fresh tissue was obtained from 16-18 week old human fetuses that had been induced by prostaglandin for therapeutic termination. The proximal tibiae were dissected free and placed in Eagle's minimal essential medium (Flow Laboratories, UK), snap frozen in liquid nitrogen, and stored at $-70^{\circ} \mathrm{C}$. Undecalcified cryostat sections $(5 \mu \mathrm{m})$ were cut, air dried at room temperature for 20 minutes, fixed in acetone at room tempera-

Table 2

\begin{tabular}{llllll}
\hline & Factors \\
\cline { 2 - 6 } & $C 1 q(m g / l)$ & Factor $B(m g / l)$ & $C 3(g / l)$ & $C 4(g / l)$ & $C 3 d(m g / l)$ \\
\hline Serum alone & $104(10)^{\star}$ & $290(34)$ & $1 \cdot 11(0 \cdot 15)$ & $0.32(0 \cdot 04)$ & $24(7)$ \\
$\%$ mean (SD) reduction in factor level after incubation & with hydroxyapatite: & \\
$10 \mathrm{mg} / \mathrm{l} \mathrm{HAC}$ & $10(1)$ & $4(2)$ & $7(2)$ & $9(3)$ & $4(4)$ \\
$100 \mathrm{mg} / \mathrm{l} \mathrm{HAC}$ & $54(6)$ & $27(2)$ & $38(4)$ & $32(4)$ & $4(4)$ \\
\hline ॠMean (SD). & & & & &
\end{tabular}

Table 3

\begin{tabular}{lllll}
\hline Serum plus: & Nil & Zymosan $10 \mathrm{mg} / \mathrm{ml}$ & $H A C 10 \mathrm{mg} / \mathrm{ml}$ & $H A C 100 \mathrm{mg} / \mathrm{ml}$ \\
\hline $10 \%$ saline & $1 \cdot 0^{\star}$ & $4 \cdot 8(0 \cdot 5)^{\dagger}$ & $1 \cdot 5(0 \cdot 1)$ & $0 \cdot 6(0 \cdot 1)$ \\
$10 \% 0 \cdot 2 \mathrm{M}$ EDTA & $1 \cdot 0$ & $1 \cdot 2(0 \cdot 1)$ & $1 \cdot 1(0 \cdot 1)$ & $0 \cdot 6(0 \cdot 1)$ \\
\hline
\end{tabular}

${ }^{\star} \mathrm{C} 3 \mathrm{~d}$ concentrations are expressed as unity in sera incubated alone.

†C3d concentrations in sera incubated with zymosan or HAC are presented as a ratio to that value for sera incubated alone. ture for 20 minutes, air dried at room temperature for 20 minutes, and stored at $-20^{\circ} \mathrm{C}$ in airtight wrapping ready for use. Further tibiae were routinely fixed and processed for paraffin wax embedding without prior decalcification. Undecalcified $5 \mu \mathrm{m}$ thick sections were cut from paraffin wax embedded tissue. The monoclonal antibodies used in this study are listed in table 1. All immunohistochemical staining of the tissue sections was done using the alkaline phosphatase-antialkaline phosphatase (APAAP) technique with no serum added at any stage. Levamisole (Sigma Chemical Co, Poole, Dorset, England) was used to block endogenous alkaline phosphatase activity. All primary antibodies were used at a range of dilutions from 1 in 5 to 1 in 500 and all results were repeated twice at optimal primary antibody concentrations. Negative controls were performed with all immunostaining procedures by using an irrelevant primary antibody (anti-rotavirus monoclonal antibody, gift of Dr CM Bunce, Department of Immunology, Birmingham University), and also by omitting primary antibody.

Alkaline phosphatase enzyme histochemistry was performed on frozen sections. Sections were incubated for 20 minutes at $37^{\circ} \mathrm{C}$ in TRIS buffer $(0.01 \mathrm{M}, \mathrm{pH} 8.2)$ containing naphthol AS-MX phosphate (Sigma) and Fast Red TR (Sigma).

Tartrate resistant acid phosphatase (TRAP) activity was demonstrated on frozen sections. Staining for TRAP and acid phosphatase was performed by incubating sections for 60 minutes at $37^{\circ} \mathrm{C}$ in an acetate buffer $(0 \cdot 1 \mathrm{M}$ sodium acetate, $\mathrm{pH} 5 \cdot 0)$ containing naphthol AS-MX phosphate (Sigma) and tetrazotised dianisidine (Fast Blue B salt, Sigma) in the presence and absence of 10 $\mathrm{mM} \mathrm{L}(+)$ sodium tartrate (Sigma). Frozen sections of fresh human liver were used as controls. The Kupffer cells stained positively for acid phosphatase, but this staining was abolished by adding $10 \mathrm{mM} \mathrm{L}(+)$ sodium tartrate to the reagent solution.

\section{Results}

\section{INCUBATION OF HYDROXYAPATITE CRYSTALS}

WITH FRESH SERUM

Fresh serum was incubated at room temperature either alone or with $10 \mathrm{mg} / \mathrm{ml}$ or 100 $\mathrm{mg} / \mathrm{ml}$ hydroxyapatite crystals (HAC); tubes were vortexed at 10 minute intervals. After 1 hour serum was separated by centrifugation and complement activation stopped by the addition of EDTA to a concentration of $0.02 \mathrm{M}$. The experiment was performed on six different serum samples and the results of subsequent immunoassays for a variety of factors are shown in table 2 .

This experiment was repeated using both fresh serum and serum heat inactivated by incubation at $58^{\circ} \mathrm{C}$ for 1 hour; these pairs of sera samples were then incubated with HAC in the presence of $10 \%$ physiological saline or $10 \% 0 \cdot 2 \mathrm{M}$ EDTA. Neither heat inactivation nor the presence of EDTA made any differ- 


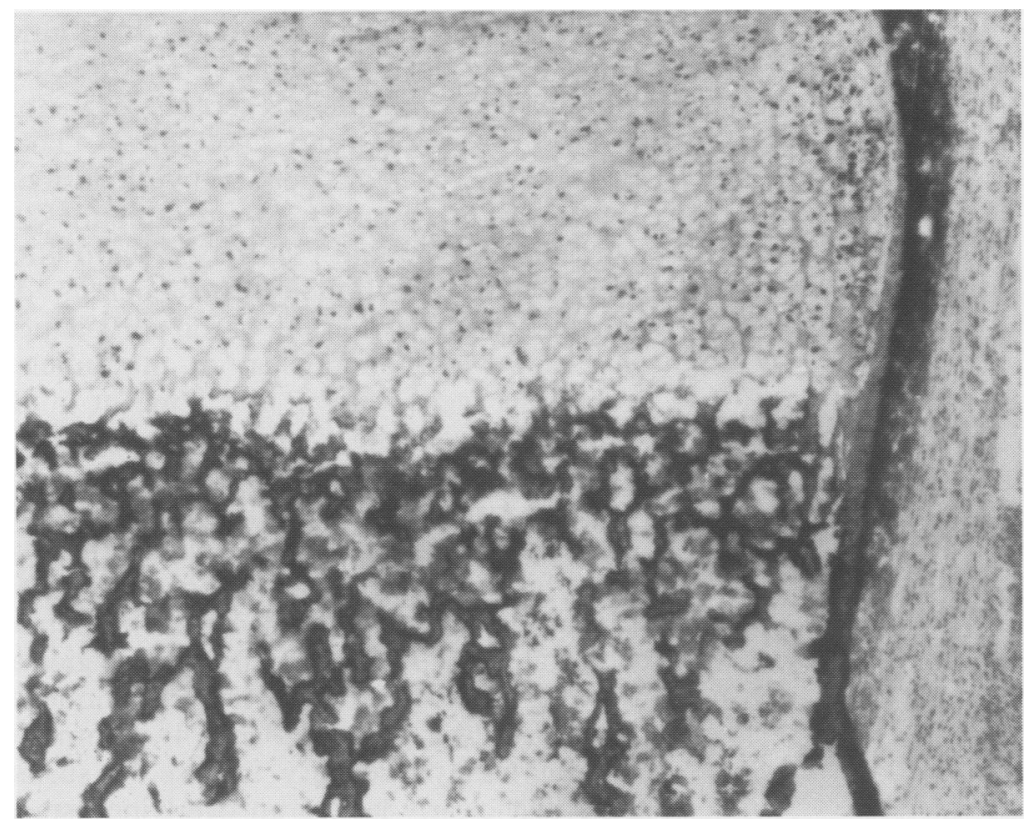

Figure 1 Human fetal endochondral growth plate and primary spongiosa. The primary spongiosa is intensely stained for alkaline phosphatase (counterstained with haematoxylin).
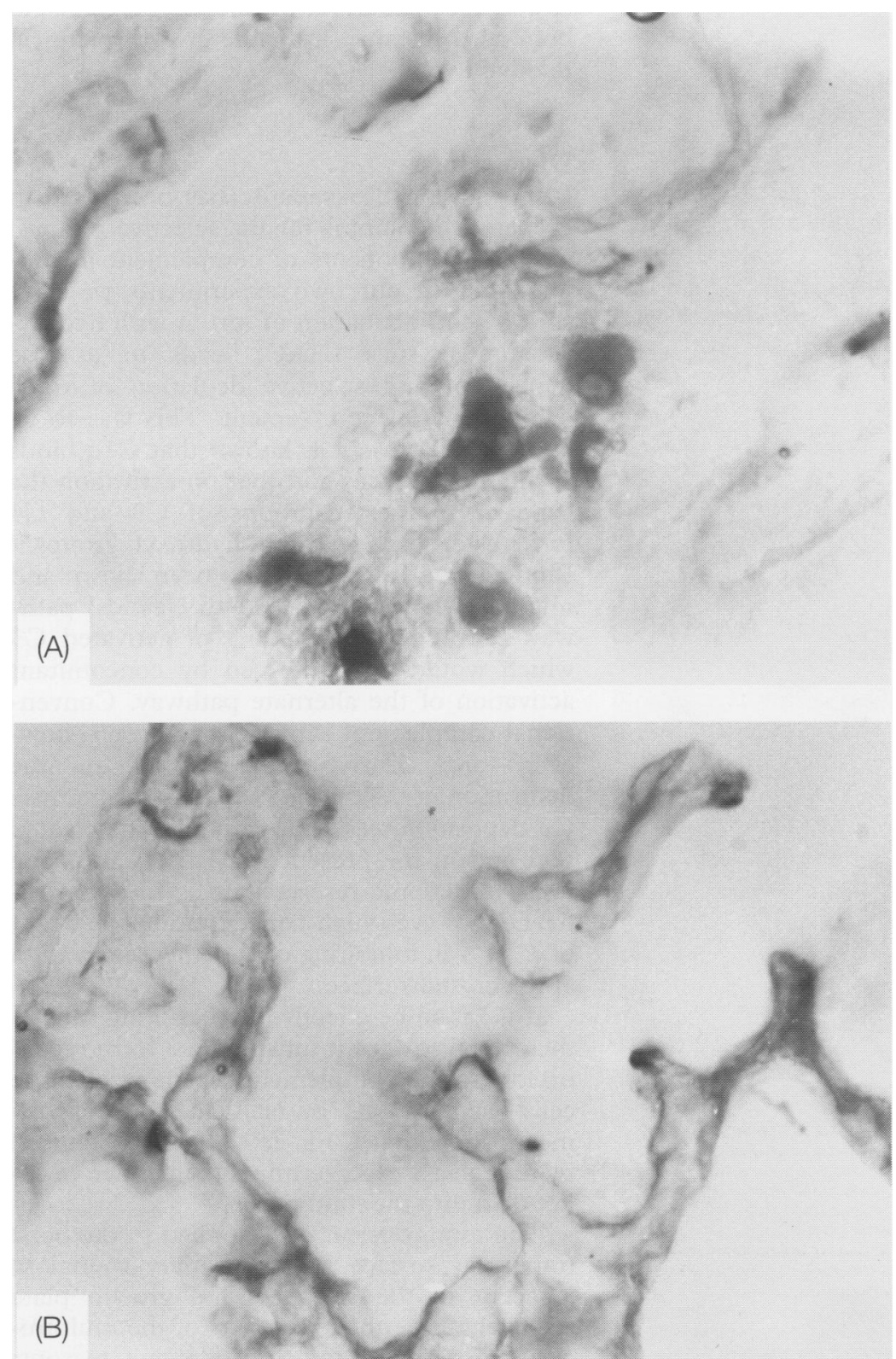

Figure 2 (A) Human fetal primary spongiosa. Tartrate resistant acid phosphatase positive multinucleate osteoclasts (no counterstain). (B) Human fetal primary spongiosa. Tartrate resistant acid phosphatase positive mononuclear cells closely applied to the bone trabecular surface (no counterstain). ence to the depletion of these complement factors by incubation with HAC (data not shown), implying that complement activation was not needed for the depletion process.

Similar serum depletion of complement factors was seen after incubation with HAC at $37^{\circ} \mathrm{C}$, with or without the presence of EDTA, over periods of up to 3 hours. Subsequent testing of incubated serum sample total haemolytic activity (plate $\mathrm{CH} 50$ assay) or alternate pathway haemolytic activity (plate AP-CH50 assay) showed an HAC dose dependent reduction in activity. This reduction in haemolytic activity was not as great as that seen after incubation with identical concentrations of the powerful alternate pathway activator zymosan, which may reflect relative preservation of $\mathrm{C} 5$ to $\mathrm{C} 9$ components, though this requires verification (data not shown).

To see if complement activation was occurring at the crystal surface in addition to what seems to be an adsorptive process of early complement components, we assayed production of the complement breakdown product C3d. Table 3 shows the results for free $\mathrm{C} 3 \mathrm{~d}$ concentrations in six serum samples after incubation with HAC with or without the complement activation inhibitor EDTA (1 hour at $37^{\circ} \mathrm{C}$ ). As expected, zymosan induced complement activation with release of free C3d into the fluid phase; this activation was inhibited by the presence of EDTA. Similar results are seen for $\mathrm{HAC}$ at $10 \mathrm{mg} / \mathrm{ml}$, though the degree of activation was not apparently as great as that seen for zymosan. $\mathrm{HAC}$ at $100 \mathrm{mg} / \mathrm{ml}$ actually reduced free C3d concentrations which we believe reflects adsorption of free C3d and lack of release of newly formed C3d from the crystal surface.

\section{IMMUNOHISTOCHEMISTRY AND ENZYME HISTOCHEMISTRY}

Haematoxylin and eosin staining of the frozen and paraffin wax sections allowed the osteoclast rich primary spongiosa to be identified. Most mononuclear cells in this region were alkaline phosphatase positive-that is, osteoblasts (fig 1). In the sections stained for TRAP, CD68, and CD61, as well as positively staining multinucleate osteoclasts, scattered mononuclear cells also stained strongly (figs 2A and B). These mononuclear cells were closely applied to the bone trabecular surface in the primary spongiosa, immediately adjacent to the endochondral growth plate, and are the mononuclear osteoclasts referred to in table 3. Positive staining of myeloid precursor cells and megakaryocytes in the haemopoietic marrow acted as an internal positive control for CD68 and CD61, respectively.

Table 1 shows a summary of the results of the immunostaining. All the positive staining results were supported by lack of staining using the negative controls described. CD68 (gp 110) and CD61 ( $\beta 3$ chain of the integrin, vitronectin receptor) positive mononuclear cells were present in similar numbers and in a similar distribution to TRAP positive mononuclear cells. A similar number of 


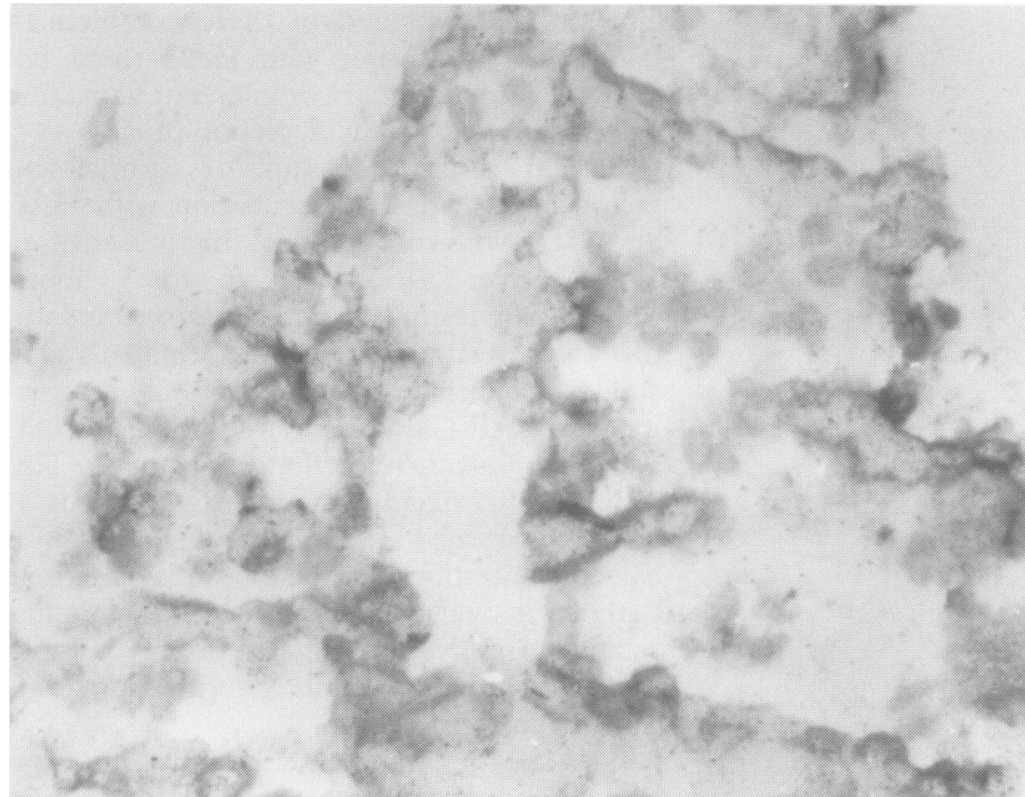

Figure 3 Human fetal primary spongiosa. CD18 positive mononuclear cells closely applied to the bone trabecular surface (no counterstain).
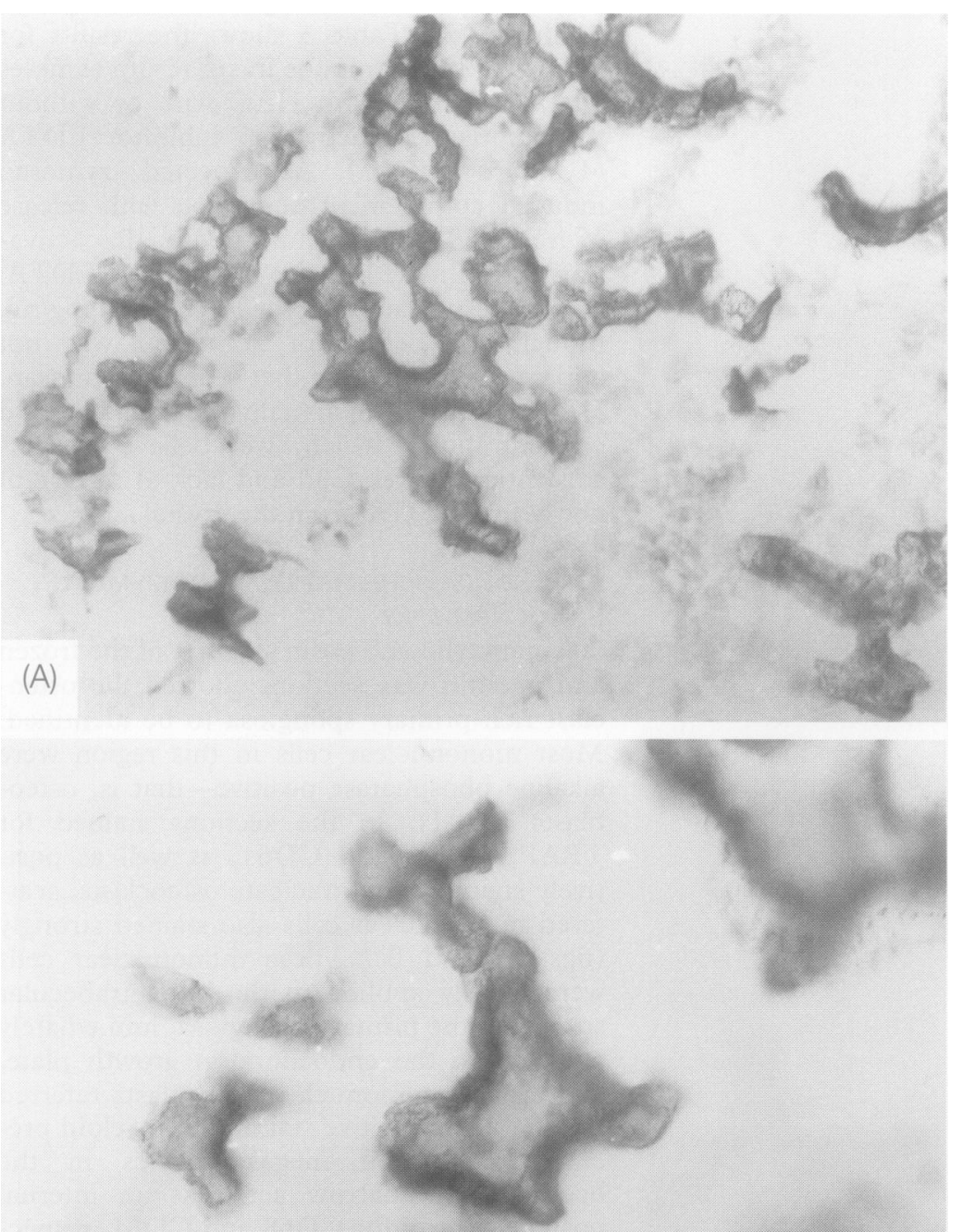

Figure 4 (A) and (B) Human fetal primary spongiosa. Focal, linear deposition of $C 3$ on the surface of the bone trabeculae: (a) low power (b) high power (no counterstain). mononuclear cells in a similar distribution also stained positively for CD11b, CD11c, and CD18 (complement receptors for $\mathrm{iC} 3 \mathrm{~b}$ also known as CR3 and CR4)(fig 3). These mononuclear cells did not stain with antibodies against the complement receptors for $\mathrm{C} 3 \mathrm{~b}$ and C3d-CD35 and CD21 (CR1 and CR2, respectively). Multinucleate osteoclasts did not stain positively for any of the complement receptors. No mononuclear or multinuclear osteoclasts were seen in the bone at a distance away from the ossification front (in the diaphysis).

Immunostaining for C3 showed a focal intense staining distribution along stretches of newly formed bone trabeculae in the primary spongiosa immediately adjacent to the endochondral growth plate (in the same region as most of the TRAP positive mononuclear osteoclasts were found) (fig 4). Immunostaining was particularly intense in surface areas that clearly lacked an overlying osteoid seam (areas where mineralised bone is exposed at the surface). C3 was not demonstrable on the surface of thicker trabeculae of bone at a distance from the growth plate (in the diaphyses).

\section{Discussion}

In the past hydroxyapatite has been used in preparative columns for the selective removal of some components of complement including C3. ${ }^{12}$ In our own experiments we have shown that incubation of serum with hydroxyapatite crystals (HAC) leads to a dose dependent and selective depletion of some components of complement. This was to be expected because it is known that $\mathrm{Clq}$ binds to anionic surfaces and that on activation the exposed thiol-ester groups of $\mathrm{C} 3$ and $\mathrm{C} 4$ form ester linkages with hydroxyl groups. ${ }^{13}$ Binding of $\mathrm{Clq}$ to HAC has been shown and might be expected to lead to classical pathway activation and binding of activated C3 which would be reinforced by concomitant activation of the alternate pathway. Conventional complement activation pathways, however, are clearly not essential to the deposition of $\mathrm{C} 3$ on the HAC surface because C3 deposition was seen with heat inactivated sera and in the presence of EDTA. But one must have some reservations as to the effectiveness of even high concentrations $(0.02 \mathrm{M})$ of EDTA in inhibiting complement activation at the crystal surface.

If $\mathrm{C} 3$ is to be effective in recruiting cells to the crystal surface it must be in a form recognisable to the complement receptors of those cells-as C3b, iC3b, or C3d. Our experiments show that $\mathrm{C} 3$ is activated as evidenced by the release of its terminal breakdown product C3d into the fluid phase.

The mononuclear cells applied to the bone trabecular surface in the primary spongiosa adjacent to the endochondral growth plate are likely to be the precursors of the multinucleate osteoclasts for the following reasons: (1) Multinucleate osteoclasts are known to form by the fusion of mononuclear precursors 
and the zone in which these mononuclear cells were identified in considerable numbers is adjacent to, and destined to become, the multinucleate osteoclast rich zone in the metaphysis. (2) The mononuclear cells described are closely applied to bone. (3) The mononuclear cells are CD68, CD61( $\beta 3$ integrin chain), and TRAP positive-markers of the multinucleate osteoclast. ${ }^{1714}$

We have shown that synthetic hydroxyapatite crystals have the ability to bind C3. That this also occurs in vivo is supported by the positive immunostaining for $\mathrm{C} 3$ on the trabecular surface of bone that is rapidly remodelling in the primary spongiosa. A similar distribution of $\mathrm{C} 3$ has been reported in mice. ${ }^{18}$ Immunostaining for C3 was most strong in foci lacking an osteoid seam, suggesting a greater deposition of $\mathrm{C} 3$ on exposed mineral surfaces. Relatively slowly metabolising diaphyseal bone showed no surface immunostaining for $\mathrm{C} 3$. We understand that the monoclonal antibody used (Dako, UK; clone HAV 3-4; Catalogue No M836) recognises an epitope that is present on both whole C3 and its larger breakdown products including $\mathrm{iC} 3 \mathrm{~b}$.

It was in the zone of $\mathrm{C} 3$ deposition that we found complement receptor positive mononuclear cells in close apposition to the bone trabecular surface. We were unable to show the presence of CR1 (CD35) or CR2 (CD21) on either the TRAP positive mononuclear cells or the multinucleate osteoclasts. CR1 and CR2 bind complement components $\mathrm{C} 3 \mathrm{~b}$ and $\mathrm{C} 3 \mathrm{~d}$, respectively. The TRAP positive mononuclear cells expressed CR3 and CR4 $(\mathrm{CD} 11 \mathrm{~b}+\mathrm{CD} 18$, and CD11c + CD18, respectively). CR3 and CR4 belong to the $\beta 2$ family of integrins (as defined by their $\beta$ chain CD18). Both receptors recognise the ArgGly-Asp (RGD) sequence of $\mathrm{iC} 3 \mathrm{~b}$ - that is, the cleaved, inactivated, and short-lived surface bound component of $\mathrm{C} 3 .{ }^{13}$ It seems reasonable to hypothesise that, in the primary spongiosa at the endochondral ossification front, $\mathrm{C} 3$ is bound to exposed HACs of newly formed bone, converted to iC $3 \mathrm{~b}, \mathrm{C} 3 \mathrm{~d}, \mathrm{~g}$ and $\mathrm{C} 3 \mathrm{~d}$, and that mononuclear precursors of osteoclasts are bound to these areas by their complement receptors. Chambers showed that in vitro macrophages fuse by the simultaneous phagocytosis of material and, in so doing, internalise and lose the surface expression of the receptors responsible for the binding of that material. ${ }^{15}{ }^{16}$ In a similar way we suggest that the mononuclear osteoclast precursors are attracted to the mineralised bone surface by binding complement and that subsequent internalisation of complement receptors 3 and 4, accounts for their absence on multinucleate osteoclasts.

This work was supported by a grant from the National Association for the Relief of Paget's Disease.

This study was the winner of the 1992 Association of Clinical Pathologists Research Award.

1 Nijweide P J, Burger E H, Feyen J H M. Cells of bone: Proliferation, differentiation, and hormonal regulation. Physiol Rev 1986;66:855-86.

2 Kahn A J, Simmons D J. Investigations of cell lineage in bone using a chimaera of chick and quail embryonic tissue. Nature 1975;258:325-7.

3 Jotereau F V, Le Douarin N M. The developmental relationship between osteocytes and osteoclasts, a study using the quail-chick nuclear marker in endochondral ossification. Dev Biol 1978;63:253-65.

4 Gothlin G, Ericsson J L E. On the histogenesis of the cells in fracture callus. Electron microscopic autoradiographic observations in parabiotic rats and studies on labelled monocytes. Virchows Arch Cell Pathol 1973; 12:318-25.

5 Walker D G. Control of bone resorption by haemopoietic tissue. ₹ Exp Med 1975;142:253-65.

6 Ash P, Loutit J F, Townsend K M S. Osteoclasts derived from haemopoietic stem cells. Clin Orthop 1981;155: 249-58

7 Athanasou N A, Quinn J. Immunophenotypic differences between osteoclasts and macrophage polykaryons: immunohistological distinction and implications for osteoclast ontogeny and function. F Clin Pathol 1990; 43:997-1004.

8 Athanasou N A, Puddle B, Quinn J, Woods C G. Use of monoclonal antibodies to recognise osteoclasts in routinely processed bone biopsy specimens. $\mathcal{f}$ Clin Pathol 1991;44:664-6.

9 Chambers T J, Fuller K. Bone cells predispose bone surfaces to resorption by exposure of mineral to osteoclastic contact. $\mathcal{F}$ Cell Sci $1985 ; 76: 155-65$.

10 Hamilton J A, Lingelbach S R, Partridge N C, Martin T J. Stimulation of plasminogen activator in osteoblast-like cells by bone-resorbing hormones. Biochem Biophys Res Comm 1984;122:230-6.

11 Heath J, Meikle M C, Atkinson S J, Reynolds J J. A factor synthesized by rabbit periosteal fibroblasts stimulates bone resorption and collagenase production by connective tissue cells in vitro. Biochim Biophys Acta 1984; 800:301-5.

12 Nilsson U R, Muller-Eberhard H J. Isolation of $\beta 1 \mathrm{f}$-globulin from human serum and its characterisation as the ulin from human serum and its characterisation as the 277-98.

13 Morgan BP. Complement. In Clinical aspects and relevance to disease. New York: Academic Press: 1990.

14 Zambonin-Zallone A, Teti A, Grano $M$, et al. Immunocytochemical distribution of extracellular matrix receptors in human osteoclasts: A $\beta 3$ integrin is colocalised with vinculin and talin in the podosomes of osteoclastoma giant cells. Exp Res 1989;182:645-52.

15 Chambers T J. Fusion of macrophages following simultaneous attempted phagocytosis of glutaraldehyde-fixed red cells. $\mathcal{f}$ Pathol 1977;122:71-80

16 Chambers TJ. Multinucleate giant cells. F Pathol 1978; 126:125-48.

17 Sato $\mathrm{T}$, Hong $\mathrm{M} \mathrm{H}$, Jin $\mathrm{C} \mathrm{H}$ et al. The specific production of the third component of complement by osteoblastic cells treated with 1a,25-dihydroxyvitamin D3. FEBS Letts 1991;285:21-4.

18 Jin $\mathrm{C} H$, Shinki $\mathrm{T}$, Hong $\mathrm{M} \mathrm{H}$ et al. 1a,25Dihydroxyvitamin D3 regulates in vivo production of the third component of complement (C3) in bone. Endocrinol 1992;131:2468-75. 